\title{
Influence of Geriatric Patients' Food Preferences on the Selection of Discharge Destination
}

\author{
Yasuko Fukuda $^{\mathrm{a}, \mathrm{d}}$, e, Mina Kohara ${ }^{\mathrm{b}}$, Asami Hatakeyama ${ }^{\mathrm{a}}$, Mikako Ochic, Masanobu Nakai ${ }^{\mathrm{c}}$
}

\begin{abstract}
Background: The nonprotein calorie/nitrogen (NPC/N) ratio of food remains poorly investigated. Thus, this study examined the nutritional factors that influence the choice of discharge destination for geriatric patients.

Methods: We retrospectively investigated the patient characteristics, clinical laboratory test results, and hospital food consumption of 65 geriatric patients $(80.0 \pm 8.2$ years; 31 males, 34 females $)$, who were receiving oral nutritional support at a small mixed-care hospital and further explored their discharge destinations. The NPC/N ratios were calculated according to the menus for the meals provided during the first 4 weeks after admission. For logistic regression analysis, the objective variables were discharge destinations (i.e., nursing care facilities including home or medical institutions) whereas the predictor variables were age, sex, nursing care level, hospitalization duration, serum albumin level (Alb), estimated glomerular filtration rate (eGFR), and $\mathrm{NPC} / \mathrm{N}$ ratio.
\end{abstract}

Results: Compared with age and nursing care level, sex (partial regression coefficient $(\mathrm{B})=-5.140, \mathrm{P}=0.002)$, hospitalization duration $(\mathrm{B}=0.077, \mathrm{P}=0.004), \mathrm{Alb}(\mathrm{B}=3.223, \mathrm{P}=0.013)$, eGFR $(\mathrm{B}=-0.071$, $\mathrm{P}=0.019)$, and $\mathrm{NPC} / \mathrm{N}$ ratio $(\mathrm{B}=-0.224, \mathrm{P}=0.001)$ are significantly correlated with the selection of discharge destination.

Conclusions: For geriatric patients who went to medical institutions, the need for prolonged hospitalization, male sex, hospitalization duration, stable serum Alb, low eGFR, low NPC/N ratio (i.e., high protein

Manuscript submitted September 2, 2020, accepted September 29, 2020

Published online November 3, 2020

${ }^{a}$ Department of Food Science and Nutrition, School of Human Environmental Sciences, Mukogawa Women's University, Ikebiraki-cho, Nishinomiya, Hyogo Prefecture 663-8558, Japan

${ }^{b}$ Formerly Affiliated With the Department of Nutrition, Nakai Hospital, Nadaku, Kobe, Hyogo Prefecture 657-0833, Japan

${ }^{\mathrm{c}}$ Department of Nutrition, Nakai Hospital, Nada-ku, Kobe, Hyogo Prefecture 657-0833, Japan

${ }^{\mathrm{d}}$ Research Institute for Nutrition Sciences, Mukogawa Women's University, Ikebiraki-cho, Nishinomiya, Hyogo Prefecture 663-8558, Japan

${ }^{e}$ Corresponding Author: Yasuko Fukuda, Department of Food Science and Nutrition, School of Human Environmental Sciences, Mukogawa Women's University, 6-46, Ikebiraki-cho, Nishinomiya, Hyogo Prefecture 663-8558, Japan. Email: yafukuda@mukogawa-u.ac.jp

doi: https://doi.org/10.14740/jocmr4337 proportion), and the quantity of hospital food consumed were the possible factors that influence their discharge destination.

Keywords: Discharge planning; eGFR; Follow-up; Hospital meal; $\mathrm{NPC} / \mathrm{N}$

\section{Introduction}

As people age, they tend to become less sensitive to hunger, indicating a diminishing appetite. Especially for the elderly during hospitalization, factors such as the etiology and pharmaceutical effects come into play, thus requiring prolonged hospital stay, although their condition becomes stable after completing treatment in the acute healthcare facility. In some cases, meal quantity and frequency can become irregular. This situation increases the risk of deficiency in energy or protein $[1,2]$.

The nonprotein calorie/nitrogen $(\mathrm{NPC} / \mathrm{N})$ ratio indicates the balance between protein and energy intake. Upsetting this balance carries the risk of affecting proper metabolism; hence, maintaining an appropriate balance between protein and energy when determining their required amounts is crucial [3].

Community-based integrated care wards at small, local mixed-care hospitals provide ongoing treatment and rehabilitation for patients who have undergone acute rehabilitation or are awaiting admission into a nursing care facility with the aim of assisting patient discharge, preferably supported by a multidisciplinary team $[4,5]$. When a patient is hospitalized, a registered dietician conducts a nutritional assessment and provides hospital meals. Appropriate nutritional support promotes patient discharge, thereby making it fundamental for the discharge process.

Thus, this study aimed to investigate the nutritional factors that influence the selection of discharge destination for geriatric patients admitted in a hospital, focusing on the estimated $\mathrm{NPC} / \mathrm{N}$ ratios of the meals that the patients consumed while being hospitalized.

\section{Materials and Methods}

\section{Subjects}

The subjects included 65 geriatric patients $(80.0 \pm 8.2$ years; 31 males, 34 females) who were admitted in a small mixed- 


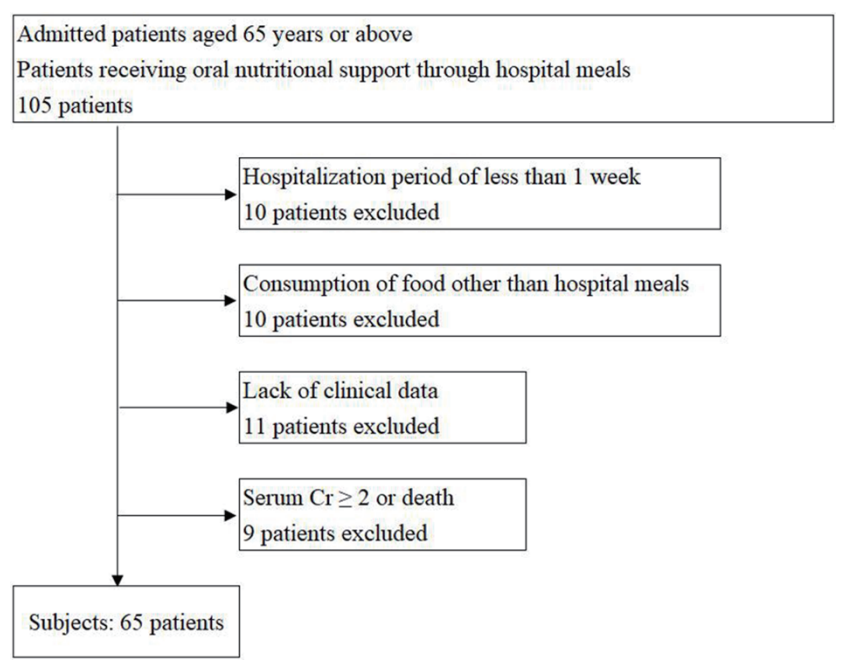

Figure 1. Flow diagram of the selection criteria for patients. Cr: creatinine.

care hospital (48 beds) in Nada Ward, Kobe City, Hyogo Prefecture, and who had received oral nutrition through hospital meals. Figure 1 illustrates a flow diagram of the selection criteria. No double-counting transpired. However, we excluded those who had severe restrictions on protein intake $(\leq 45 \mathrm{~g} /$ day). Then, we retrospectively extracted basic patient attributes from medical records and explored their discharge destinations. The study period was from April 2019 to July 2020.

The included attributes were age during admission, sex, height, weight, comorbidity state, serum albumin level (Alb), blood glucose level, blood urea nitrogen, serum creatinine, estimated glomerular filtration rate (eGFR), nursing care level, hospitalization duration in days, discharge destination, hospital meal type provided, and hospital meal consumption pattern.

We calculated the body mass index (BMI) using the height and weight and scored the comorbidities according to the Carlson Comorbidity Index (CCI) [6].

Nursing care levels (from 1 (assistance required) to 5 (nursing care required)) were re-scored on a scale of 1 to 7 . Meanwhile, hospitalization duration was defined as the number of days from admission until discharge.

Furthermore, the discharge destination was investigated to determine whether it was a nursing care facility or a medical institution. Medical institutions included either hospitals or nursing and healthcare facilities for the elderly. "Home discharge" was categorized under nursing care facilities. [7]. All of the subjects underwent a structured rehabilitation program.

To assess the subjects' consumption of hospital food, we identified their consumption of main and side dishes during the first 4 weeks of hospitalization, as described in their medical records. The nutritional content of these dishes was estimated according to their corresponding menus. After quantifying their energy and protein content, we calculated their NPC/N ratios by using the following formula: (energy from carbohydrates and fats (in calories) $) /($ protein $($ in grams $) \times 0.16)=$ $\mathrm{NPC} / \mathrm{N}$ ratio.

We selected these factors for the predictor variables based on the following reasons: 1) level of nursing care is an indicator of a level of physical function; 2) duration of hospitalization is an indicator of treatment efficacy (i.e., whether further treatment is required); 3) Alb is an indicator of nutritional status; 4) eGFR is an indicator of organ function status with aging; and 5) NPC/N is an indicator of evaluating diet bias and nutritional balance.

\section{Statistical analysis}

\section{Evaluation of NPC/N ratios}

The NPC/N ratio stated in the hospital dietary rules $(\mathrm{NPC} / \mathrm{N}$ ratio before eating) and the estimated $\mathrm{NPC} / \mathrm{N}$ ratio based on food consumption (NPC/N ratio after eating) were assessed by paired $t$-test.

\section{Search for factors that influence discharge destination}

In conducting logistic regression analysis, we considered nursing care facilities or medical institutions as the objective variables, and age, sex, level of nursing care, duration of hospitalization, serum Alb, eGFR and NPC/N ratio were considered as the predictor variables. For the NPC/N ratio, we determined the NPC/N ratio before and after eating and compared the results.

All statistical data were analyzed using the Statistical Analysis System (SAS) version 9.4 (SAS Institute Inc., Cary, $\mathrm{NC}$, USA), and the significance was determined by two-tailed test in which $\mathrm{P}<0.05$ was considered to be statistically significant.

\section{Ethical considerations}

The Research Ethics Committee of Mukogawa Women's University and Junior College approved this study on March 15, 2019 (Approval No. 18-85). In accordance with the Declaration of Helsinki, the study content was explained to the subjects beforehand and those who consented to participate were included in the study. We also secured a consent to use their sensitive, personal information while maintaining anonymity during the analysis.

\section{Results}

\section{Subject demographics}

As shown in Table 1, the main conditions included chronic heart failure, bronchial pneumonia, chronic obstructive pulmonary disease, type 2 diabetes, lumbar compression fracture, hypertension, hyperlipidemia, femoral neck fracture, Parkinson's disease, disuse-related atrophy of the lower-limb muscles, and degenerative lumbar spondylosis. Meanwhile, comorbidities 
Table 1. Demographics

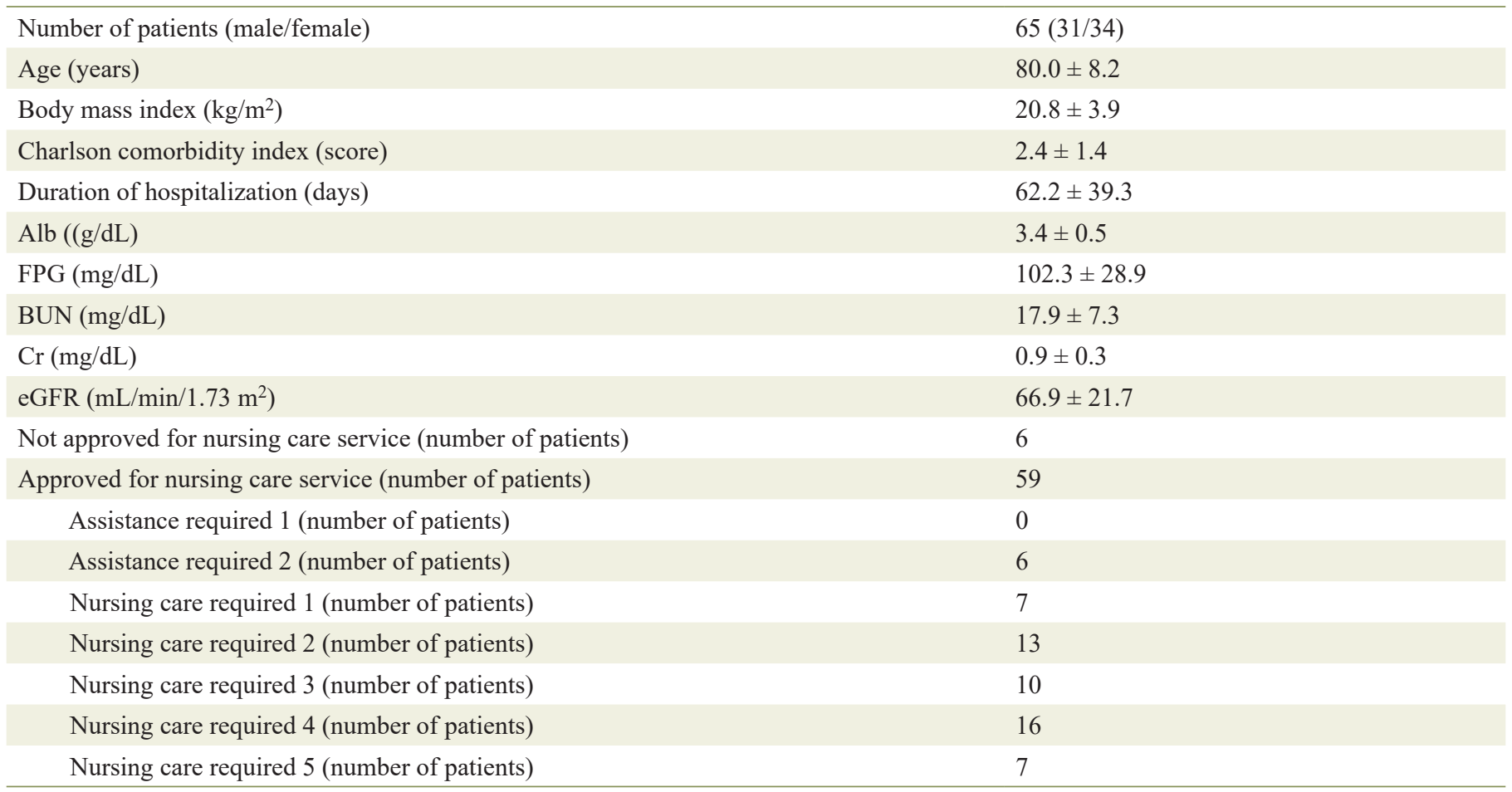

Data are expressed as mean \pm standard deviation. The main diseases for admission included chronic heart failure, bronchial pneumonia, chronic obstructive pulmonary disease, and type 2 diabetes; however, these conditions had been treated in the acute care hospital, and thus, patients' condition became stable. Alb: serum albumin; FPG: fasting plasma glucose; BUN: blood urea nitrogen; Cr: creatinine; eGFR: estimated glomerular filtration rate.

included chronic hepatitis $\mathrm{B}$, chronic hepatitis $\mathrm{C}$, type 2 diabetes, lipid abnormality, gastric ulcer, iron-deficiency anemia, and constipation. The main conditions and comorbidities had been treated at the acute care hospital, and thus the patients' conditions became stable.

\section{$\mathrm{PC} / \mathrm{N}$ ratio}

As shown in Table 2, the average before eating NPC/N was $138 \pm 11$ (maximum value: 148; minimum value: 118 ), whereas the average after eating $\mathrm{NPC} / \mathrm{N}$ was $135 \pm 17$ (maximum value: 218; minimum value: 103 ).

Hospital meals that were ordered for patients were as follows: 17 cardio/hypertension meals (26.2\%), 15 diabetes meals (23.1\%), 14 normal meals $(21.6 \%), 8$ liver meals $(12.3 \%), 6$ lipid abnormality meals $(9.2 \%), 3$ kidney meals (4.6\%), 1 ulcer meal (1.5\%), and 1 anemia meal (1.5\%).

\section{Investigation of factors that influence discharge destination}

Nursing care facilities were selected for 51 patients, whereas medical institutions were selected for 14 patients.

Table 2. Hospital Food Consumption

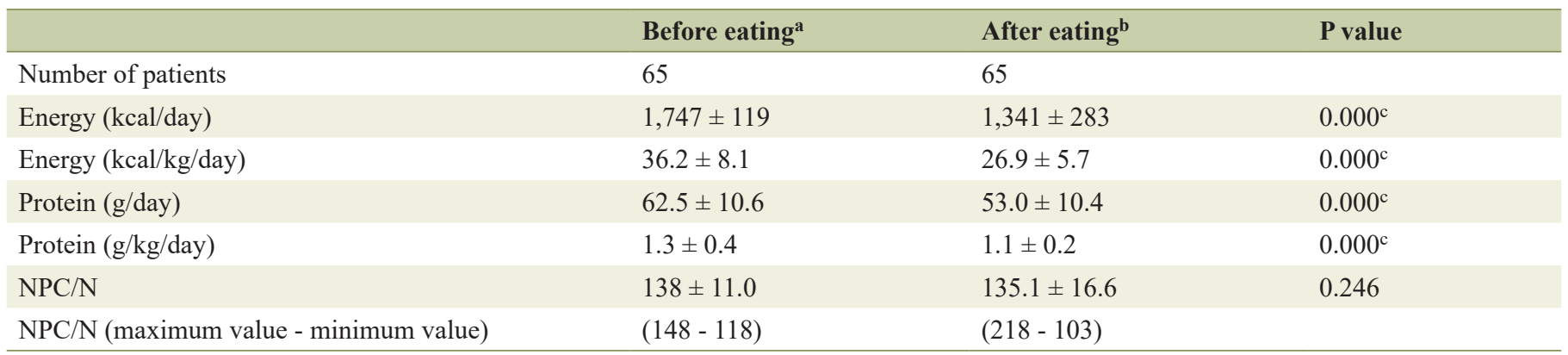

aCalorie intake calculated from the menu served to patients. ${ }^{b}$ Calorie intake calculated from actual food consumption. ${ }^{\mathrm{CP}}<0.001$, paired $t$-test. Data are expressed as mean \pm standard deviation. NPC/N: nonprotein calorie/nitrogen. 
Table 3. Factors That Influence Discharge Destination Extracted Using the NPC/N Ratio Calculated From the Actual Food Consumed $\left(\mathrm{NPC} / \mathrm{N}^{\mathrm{a}}\right)$

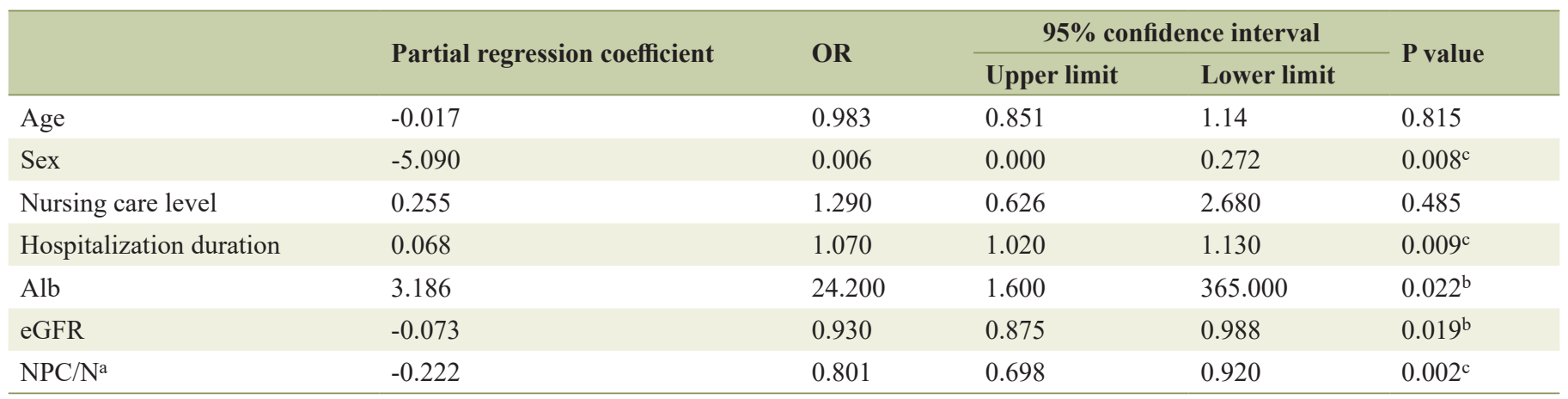

aNPC/N of food consumed, $n=65$, logistic regression analysis. ${ }^{b} \mathrm{P}<0.05$. ${ }^{\mathrm{c}} \mathrm{P}<0.01$. OR: odds ratio; Alb: serum albumin; eGFR: estimated glomerular filtration rate; NPC/N: nonprotein calorie/nitrogen.

Table 4. Factors That Influence Discharge Destination Extracted Using the NPC/N Ratio Calculated From the Hospital Meal Menu $\left(\mathrm{NPC} / \mathrm{N}^{\mathrm{a}}\right)$

\begin{tabular}{|c|c|c|c|c|c|}
\hline & Dactiol moration & OD & $95 \%$ co & nce interval & D wolno \\
\hline & Fartial regression coemeitiot & ON & Upper limit & Lower limit & Pvalue \\
\hline Age & -0.082 & 0.921 & 0.809 & 1.050 & 0.211 \\
\hline Nursing care level & 0.140 & 1.150 & 0.627 & 2.100 & 0.654 \\
\hline Hospitalization duration & 0.020 & 1.020 & 0.986 & 1.060 & 0.243 \\
\hline $\mathrm{NPC} / \mathrm{N}$ & 1.541 & 4.670 & 0.000 & 0.000 & 0.995 \\
\hline
\end{tabular}

${ }^{a} \mathrm{NPC} / \mathrm{N}$ calculated from hospital meal menu, $\mathrm{n}=65$, logistic regression analysis. OR: odds ratio; Alb: serum albumin; eGFR: estimated glomerular filtration rate; NPC/N: nonprotein calorie/nitrogen.

\section{Analysis using the NPC/N ratio after eating}

Discharge destination had a significantly positive correlation with hospitalization duration or serum Alb but had a significantly negative correlation with sex, eGFR, or consumed NPC/N (Table 3). Most patients who went to nonhome destinations were males, had long hospitalization duration, and did not have a low serum Alb level, but they had low eGFR and a low NPC/N ratio. Variables such as age and nursing care level did not influence discharge destination.

\section{Analysis using the NPC/N ratio before eating}

When using the NPC/N ratio before eating, we found no variables that influenced discharge destination (Table 4, Fig. 2).

\section{Discussion}

In the present study, we quantified the energy and protein consumption of geriatric patients at a small, local mixed-care hospital, calculated the NPC/N ratios of these diets, and identi- fied dietary factors that had an influence in deciding patients' discharge destination. When the analysis included the NPC/N ratios estimated from the actual diets that patients consumed, we clearly found that the factors associated with patients being discharged to medical institutions included the need for prolonged hospitalization, the male sex, hospitalization duration, stable serum Alb, low eGFR, and a low NPC/N ratio. However,

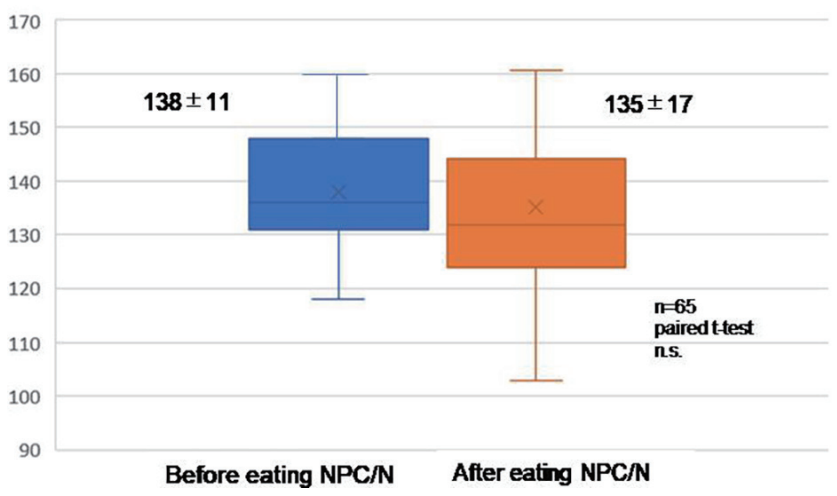

Figure 2. NPC/N before eating vs. after eating. NPC/N: nonprotein calorie/nitrogen. 
when the analysis used the NPC/N ratios calculated only from the nutritional information in the hospital dietary rules regardless of the actual quantities consumed by the patient, we found no factors that influenced the choice of discharge destination.

There was no significant difference in the mean NPC/N ratios before and after eating. However, the NPC/N ratio after eating showed a significant negative association with the objective variable (discharge destination). This was probably due to the degree of variance in both before and after eating NPC/N ratios. The maximum and minimum values of before-eating NPC/N were 148 and 118, respectively, whereas those of after-eating NPC/N were 218 and 103, respectively. These results suggest that beforeeating and after-eating NPC/N are independent variables.

The NPC/N ratio indicates the appropriate quantity of protein required to produce favorable conditions for protein synthesis in the body, and it determines the energy/protein balance based on food consumption [3]. A low NPC/N ratio means that protein has a large quantity in relation to energy. An NPC/N ratio of approximately $80-100: 1$ is desirable for patients under severe stress from severe burns/physical injury, and a ratio of $100-150: 1$ is desirable for those with physical injury. The ratio decreases when a full dose of protein is required. Conversely, the NPC/N ratio increases to over 200:1 when protein intake must be restricted because of renal failure or other conditions. An NPC/N ratio of approximately 150 - 200:1 is appropriate for patients in a stable condition as long as their renal function is normal [3]. Providing food with a low NPC/N ratio (specifically, a protein-rich concentrated liquid food) reportedly reduces mechanical ventilation time and improves the medium-term nutritional state of patients with severe emergency [8]. According to a published guideline, when managing the nutrition of newborns, providing food with an NPC/N ratio of $200-250: 1$ is necessary to optimize nitrogen utilization without stressing their immature kidney function [9].

We believed that the NPC/N ratios of the food consumed by our subjects were influenced by the type of hospital food ordered and the actual quantity consumed. Hospital meals are prepared according to patients' clinical conditions and contain protein and energy quantities specified by hospital dietary rules $[10,11]$. Thus, different meal types have different NPC/N ratios. Accordingly, the NPC/N ratio of food ingested by a patient depends on which type of meal is ordered. Moreover, the actual quantity of food a patient consumes varies according to his or her appetite and personal predilections; these two factors influence not only the quantities of protein and energy ingested but also the NPC/N ratio.

In our study, 37 out of 65 subjects (57\%) left part or all of their staple food and/or side dishes uneaten. Their reasons included "lack of appetite" and "too much rice," and the quantity of food left uneaten varied daily. Consequently, the NPC/N ratios also differed daily.

When we investigated the factors that influenced which destination a patient was discharged to and incorporated the NPC/N ratios of the food actually consumed, we clearly found that stable serum Alb levels, low eGFR, and low NPC/N ratios were the factors associated with discharge to medical institutions. When the NPC/N ratios calculated from the hospital dietary rules were used for statistical analysis, no variables were associated with discharge destinations. Therefore, even when the influence of eGFR is eliminated, the NPC/N ratio that is estimated from the actual food consumed still significantly affects the selection of discharge destination.

Serum Alb is a type of protein present in the blood, and low Alb often indicates undernutrition [12]. Protein intake is increased to improve serum Alb. Meanwhile, eGFR shows the estimated rate of filtration through the glomerulus, and a low eGFR indicates renal function decline [13]. When renal function decreases, protein intake is restricted as a part of the diet therapy [14]. A low NPC/N ratio indicates a high protein intake. When a diet is high in protein, serum Alb may improve, but the kidneys may be stressed, causing eGFR decline. In fact, $2.0 \mathrm{~g} / \mathrm{kg}$ of body weight/day can increase the risk of renal dysfunction even in healthy elderly patients [15]. A prospective cohort study that investigated elderly women with mild renal dysfunction (eGFR: $55.88 \mathrm{~mL} / \mathrm{min} / 1.73 \mathrm{~m}^{2}$ ) showed that a high protein intake (> $1.3 \mathrm{~g} / \mathrm{kg} /$ day) resulted in the deterioration of renal function [16].

In the current study, nonhome discharge positively correlated with serum Alb but negatively correlated with eGFR or NPC/N ratio. Considering that the clinical laboratory test data we used were those from the time of admission, we could not identify which variable was the cause and which one was the result. However, a causal relationship clearly existed. Therefore, the clinical condition of each geriatric patient should be paid attention to in order to facilitate discharge. According to the hospital dietary rules incorporated in this study, meals with low NPC/N ratios were for diabetes (118:1), lipid abnormality (129:1), and cardio/hypertension (134:1). Diabetes meals were ordered for 15 patients $(23.1 \%)$, and the obtained results may potentially be biased. However, diabetes meal is ordered commonly for geriatric patients with metabolic diseases as a therapeutic diet. Therefore, when patients with low eGFR are involved, hospital meals should be reviewed, and patients should be stopped from eating merely what they prefer.

The nutritional status of geriatric patients with chronic diseases may affect the selection of discharge destination. Hence, nutritional guidance should be provided while paying close attention not only to primary disease and comorbidities but also to individual characteristics, such as decreased renal function.

\section{Limitations of the study}

Our subjects were geriatric patients who were admitted at a small, local mixed-care hospital in Nada Ward, Kobe City, Hyogo Prefecture, and who could consume food orally. Hence, the sample size was small. Discharge destination is also influenced by factors other than nutritional condition. These factors include social factors (e.g., the state of their family/their ability to care), financial factor, mental or psychological factors (e.g., cognitive dysfunction/depression), and their level of independence in everyday life. Therefore, the interpretation of the results is limited.

\section{Conclusions}

The selection of discharge destination can be influenced the 
need for prolonged hospitalization, by the food preferences of geriatric patients during hospitalization, low eGFR, and meals with a low NPC/N ratio (high in protein). For geriatric patients who were continuously prescribed with low-NPC/N diabetes meals or lipid abnormality meals, the meal type must be reviewed, and they should be given nutritional guidance on how to balance energy and protein through diet.

\section{Acknowledgments}

We would like to express our gratitude for Nakai Hospital and their in-patients who agreed to participate in the study, the medical social workers Masamichi Onishi and Atsushi Ueno, and other medical staff. We also would like to express our gratitude to Prof. Teruyoshi Amagai of Mukogawa Women's University, who kindly provided guidance during the composition of the paper.

\section{Financial Disclosure}

None to declare.

\section{Conflict of Interest}

The authors have no conflicts of interest.

\section{Informed Consent}

The contents of the study were explained to the subjects in advance, and written informed consents were obtained.

\section{Author Contributions}

YF drew up the research plan, dealt with matters regarding ethical research screening, provided detailed explanation of the research to the subjects, collected/analyzed data, and prepared the initial draft. $\mathrm{MK}, \mathrm{AH}$, and $\mathrm{MO}$ collected the data. $\mathrm{MN}$ drew up the research plan and matters regarding ethical research screening. MN also explained the research to the subjects and obtained their consent. All authors agree to the submission of this paper.

\section{Data Availability}

The authors declare that the data supporting the findings of this study are available within the article.

\section{References}

1. Sanson G, Bertocchi L, Dal Bo E, Di Pasquale CL, Zanetti M. Identifying reliable predictors of protein-energy malnutrition in hospitalized frail older adults: A prospective longitudinal study. Int J Nurs Stud. 2018;82:40-48.

2. Drevet S, Gavazzi G. [Undernutrition of the elderly]. Rev Med Interne. 2019;40(10):664-669.

3. Amagai T, Hasegawa M, Kitagawa M, Haji S. Non-protein calorie: Nitrogen Ratio (NPC/N) as an indicator of nitrogen balance in clinical settings. Biomed J Sci \& Tech Res. 2018;6(1):5013-5015.

4. Chin-Jung L, Shih-Jung C, Shou-Chuan S, ChengHsin C, Jin-Jin T. Discharge planning. Int J Gerontol. 2012;4:237-240.

5. Goncalves-Bradley DC, Lannin NA, Clemson LM, Cameron ID, Shepperd S. Discharge planning from hospital. Cochrane Database Syst Rev. 2016;1:CD000313.

6. Charlson ME, Pompei P, Ales KL, MacKenzie CR. A new method of classifying prognostic comorbidity in longitudinal studies: development and validation. J Chronic Dis. 1987;40(5):373-383.

7. Ministry of Health, Labour and Welfare, the law regarding the creation of relevant laws to ensure the comprehensive promotion of community-based medical and nursing care. 2014 Law No. 83.

8. Cerra FB, Shronts EP, Raup S, Konstantinides N. Enteral nutrition in hypermetabolic surgical patients. Crit Care Med. 1989;17(7):619-622.

9. Koletzko B, Goulet O, Hunt J, Krohn K, Shamir R, Parenteral Nutrition Guidelines Working G, European Society for Clinical N, et al. 1. Guidelines on Paediatric Parenteral Nutrition of the European Society of Paediatric Gastroenterology, Hepatology and Nutrition (ESPGHAN) and the European Society for Clinical Nutrition and Metabolism (ESPEN), Supported by the European Society of Paediatric Research (ESPR). J Pediatr Gastroenterol Nutr. 2005;41(Suppl 2):S1-87.

10. Dijxhoorn DN, van den Berg MGA, Kievit W, Korzilius J, Drenth JPH, Wanten GJA. A novel in-hospital meal service improves protein and energy intake. Clin Nutr. 2018;37(6 Pt A):2238-2245.

11. Sheils JF, Rubin R, Stapleton DC. The estimated costs and savings of medical nutrition therapy: the Medicare population. J Am Diet Assoc. 1999;99(4):428-435.

12. Jabbour J, Abou Ali AN, Rabeh W, Al-Shaar L, Avgerinos ED, Habib RH. Role of nutritional indices in predicting outcomes of vascular surgery. J Vasc Surg. 2019;70(2):569-579 e564.

13. Warnock DG. Estimated glomerular filtration rate: fit for what purpose? Nephron. 2016;134(1):43-49.

14. Ko GJ, Obi Y, Tortorici AR, Kalantar-Zadeh K. Dietary protein intake and chronic kidney disease. Curr Opin Clin Nutr Metab Care. 2017;20(1):77-85.

15. Walrand S, Short KR, Bigelow ML, Sweatt AJ, Hutson SM, Nair KS. Functional impact of high protein intake on healthy elderly people. Am J Physiol Endocrinol Metab. 2008;295(4):E921-928.

16. Knight EL, Stampfer MJ, Hankinson SE, Spiegelman D, Curhan GC. The impact of protein intake on renal function decline in women with normal renal function or mild renal insufficiency. Ann Intern Med. 2003;138(6):460467. 